\title{
Moving beyond access: Towards a quality-orientated substance abuse treatment system in South Africa
}

There is growing concern about the increased demand for and limited access to substance abuse treatment in South Africa. ${ }^{1}$ The government has responded by allocating more money to the delivery of substance abuse treatment, expanding the number of state-funded treatment slots, and training additional health and social workers to deliver these services, particularly in provinces where the prevalence of substance-related problems is high, such as the Western Cape. While these efforts should be commended and continued, steps to improve service availability have occurred without adequate consideration of the quality of services provided. This is not surprising, as there is little or no routine monitoring and evaluation of substance abuse services in the country. ${ }^{2}$ It is also disquieting, as access to treatment is necessary but not sufficient for positive treatment outcomes. ${ }^{3}$

Focusing on treatment quality is especially important because of public and consumer concerns about the quality and effectiveness of substance abuse treatment and whether public money is being spent efficiently to achieve the best possible outcomes. ${ }^{4}$ Service quality is a concern too of South African policy-makers. The Third (draft) National Drug Master Plan $(2012-2016)^{5}$ and the Department of Health's Mini Drug Master Plan $(2011-2014)^{6}$ specify service quality improvement as a priority requiring action. Improving service quality is also a key focal area of the strategic framework for 2010 - 2013 of the National Department of Health, which established an Office of Health Standards Compliance in 2012 that is tasked with quality assurance activities. ${ }^{7}$

To meet this goal of improving service quality, objective data on the quality of substance abuse treatment must be routinely collected from all service providers. Such a service quality measurement system holds significant potential benefits for consumers, service providers and policy-makers. For consumers, it can provide reliable data on the effectiveness of care (which may counter negative perceptions about treatment that act as barriers to treatment use). ${ }^{4}$ Information can be generated about the expected outcomes of a particular service, allowing consumers to make informed choices about where to seek services. ${ }^{8-9}$ For service providers, objective and continuous data on the quality of their services are useful for driving evidence-based programme change. Data can identify areas in treatment services that may benefit from change, measure change in service quality over time, and provide feedback on the success of interventions to improve service quality. ${ }^{3,8-11}$ For policy-makers, when standardised measures are used to collect data from all service providers, these measures can be used to compare the performance of treatment providers and benchmark service providers against a minimum standard. ${ }^{3,9}$ This will help identify areas and strategies for system improvement and guide policy-makers and service planners in terms of where best to allocate scarce resources. ${ }^{8,10}$ Consumers can use information about programme performance to choose a suitable service provider, ${ }^{9}$ and providers can use data to advocate for better funding based on evidence of the quality of their services. ${ }^{9,11}$

Despite the potential benefits of collecting data on treatment service performance, South Africa has no data collection system suitable for this purpose. Although the South African Community Epidemiology Network on Drug Use (SACENDU) ${ }^{12}$ routinely collects data from most substance abuse treatment facilities in the country, SACENDU is epidemiological in focus and does not collect data on the quality or effectiveness of treatment. In addition, routine monitoring of service performance and treatment outcomes is rare in substance abuse treatment services. ${ }^{2} \mathrm{~A}$ few services have collected data of this kind for internal programme evaluations or as part of a small research initiative. However, as these data collection efforts have used their own purpose-constructed (and non-validated) monitoring tools and have been time-limited, they have not allowed for changes in the quality of services to be monitored or comparisons with other treatment services. Consequently, their data are not useful to assess and track the quality of services provided by the substance abuse treatment system as a whole. A purpose-driven system must therefore be developed to monitor the performance and quality of substance abuse treatment services in the country.

In developing such a system for South Africa, we can learn from the systems used to monitor the quality of addiction and mental health services in other countries. ${ }^{8-11}$ First, there must be agreement about the core data needed to make judgements about service quality. A broad range of stakeholders (treatment providers, policy-makers and service planners) should identify the main goals of the treatment system, key domains that address each of these goals and together constitute quality services, the core indicators within each domain that are feasible and important to measure, and measurement specifications for each selected indicator (known as service quality measures, SQMs)., ${ }^{3,8-11}$ Second, the system should comprise a small number of SQMs to limit additional data collection burden on treatment providers. Carefully selecting a small number of priority measures is important, as previous attempts to routinely collect treatment service data have failed largely because of lengthy forms and the collection of seemingly irrelevant information. Individual treatment services may wish to collect additional data on indicators that are relevant for their programme, and this should be encouraged. However, it is essential that the core set of SQMs are collected in a standardised way by all treatment services to facilitate the comparison and benchmarking of treatment services against a minimum standard. ${ }^{3,8-11}$ Third, there must be widespread support among treatment providers for the routine application of these SQMs as part of standard clinical care and a commitment to use the system's data to improve clinical practice. ${ }^{9-11}$ This implies that the system will provide a good mechanism for feeding back data to service providers.

\section{Current developments}

The good news is that steps have been taken to develop a service quality measurement system for South African substance treatment services. This started with the formation of a national steering committee comprising a broad range of stakeholders, including policy-makers from relevant government departments, representatives from the Central Drug Authority, treatment providers from several provinces, academics and researchers. The Medical Research Council's Alcohol and Drug Abuse Research Unit provides the co-ordination and implementation functions. The steering committee first agreed upon the main goals for the substance abuse treatment system and through consensus identified five variable domains corresponding to these goals: treatment effectiveness, treatment efficiency, access to services, person-centered services, and quality of care. The committee identified potentially useful indicators for measuring progress within each domain. A Delphi exercise assessed the relevance and importance of each indicator for the substance abuse treatment system and the feasibility of developing measurement specifications for this indicator. Based on its findings, the steering committee selected (through consensus) a core set of indicators and debated the best ways of collecting data for each indicator. The 
committee used a consumer survey and administrative data collected from service providers as modes of measurement. Consumer survey and administrative measures work groups were formed to specify SQMs for each selected indicator and support pilot testing of these SQMs. The consumer survey has been pilot-tested and psychometric validation of the measures is underway. Focus groups have been conducted with service providers to inform the development of administrative indicators and additional data elements identified to incorporate into the SACENDU data collection forms (being assessed for feasibility and acceptability).

Although this initiative holds promise, much work is required to ensure that the system is successfully implemented and that the data generated are used as intended. To ensure the successful implementation and use of the SQM system, we recommend that the consensus-driven approach adopted by this initiative be continued. It is important that broad-based buy-in is obtained from relevant stakeholders at each stage of system development and implementation so that the system remains stakeholder-driven rather than researcher-driven. The SQM system must also remain sufficiently scientifically robust to provide evidence that can be used for programme improvement and system change. We urge policymakers and providers to resist the temptation to expand the proposed set of SQMs beyond those absolutely necessary. The SQM initiative has the best chance of success and the system is more likely to generate longitudinal data if it only collects data on a minimal set of indicators, thereby limiting the burden on service providers. We recommend that the SQM initiative retain its data collection flexibility. Currently the system allows for data to be collected electronically or by hand to include facilities without electronic resources. Nonetheless, we should continue to assess how technological developments can improve and standardise data collection. We also recommend retaining the current focus on generating 'usable' data. This ensures that data allow for comparisons to be made across facilities and are accessible to providers in formats that they can easily understand and use to improve programmes. Finally, as the goal of this initiative is to ensure that the data are used to improve service quality, we strongly recommend that capacity to interpret and use the data is developed among service providers and policy-makers before system implementation and that ongoing support for data interpretation is provided to system end-users.

In conclusion, the benefits of implementing such an SQM system are likely to extend beyond improving the quality of substance abuse care in the country, as it could provide a template for service improvement initiatives in other spheres of health service delivery. Developers of the National Health Insurance (NHI) should take notice of this initiative and consider how quality measurement can be built into the monitoring of the NHI from the outset. Failure to extend the current narrow focus on improving access to health services to include a quality focus may represent a missed opportunity to improve the health of South Africans.

Acknowledgements. We thank all members of the SQM national steering committee and participating treatment facilities for their continued support and participation in this initiative.

\section{Bronwyn Myers}

Alcohol and Drug Abuse Research Unit, South African Medical Research Council, and Department of Psychiatry and Mental Health University of Cape Town

\section{Zaino Petersen}

\section{Rehana Kader}

Alcohol and Drug Abuse Research Unit South African Medical Research Council

\section{Charles D H Parry}

Alcohol and Drug Abuse Research Unit

South African Medical Research Council, and Department of Psychiatry

Stellenbosch University

Tygerberg, Western Cape

Corresponding author: B Myers (bmyers@mrc.ac.za)

1. Myers B, Louw J, Pasche S. Inequitable access to substance abuse treatment services in Cape Town, South Africa. Subst Abuse Treat Prev Policy 2010;5:28. [http://dx.doi.org/10.1186/1747-597X-5-28] 2. Myers B, Burnhams NH, Fakier N. Monitoring and evaluation of substance abuse services in South Ayers $\mathrm{B}$, Burnhams $\mathrm{NH}$, Fakier $\mathrm{N}$. Monitoring and evaluation of substance abuse services in South
Africa: implications for policy and practice. International Journal of Mental Health and Addiction Africa: implicat

3. Knott AM, Corredoira R, Kimberly J. Improving the consistency and quality of service delivery? Knott AM, Corredoira R, Kimberly J. Improving the consistency and quality of service delivery:
Implications for the addiction treatment field. I Subst Abuse Treat 2008;35:99-108. [http://dx.doi. org/10.1016/j.jsat.2007.09.005]

4. Myers B, Fakier N, Louw J. Stigma, treatment beliefs, and substance abuse treatment use in historically disadvantaged communities. African Journal of Psychiatry 2009;12:218-222. [http://dx.doi. org/10.4314/ajpsy.v12i3.48497]

5. South African Department of Social Development. Third National Drug Master Plan 2012-2016. Draft. Pretoria: Department of Social Development, 2011.

6. South African Department of Health. Mini Drug Master Plan (2011/12-2013/14). Pretoria: Department of Health, 2012

. South African Department of Health. National Department of Health: Strategic Plan (2010/11-201213). Pretoria: Department of Health, 2010

8. Garnick DW, Lee MT, Horgan CM, Acevedo A. Adapting Washington Circle performance measures for public sector substance abuse treatment systems. J Subst Abuse Treat 2009;36:265-277. [http:// dx.doi.org/10.1016/j.jsat.2008.06.008

9x.doi.org/10.1016/j.jsat.2008.06.008] McCarty D. Performance measurement for systems treating alcohol and
Abuse Treat 2007;33:353-354. [http://dx.doi.org/10.1016/j.jsat.2007.04.002]

10. Tiet QQ, Byrnes HF, Barnett P, Finney JW. A practical system for monitoring the outcomes of substance use disorder patients. J Subst Abuse Treat 2006;30:337-347. [http://dx.doi.org/10.1016/j. jsat.2006.03.002

11. Kilbourne AM, Keyser D, Pincus HA. Challenges and opportunities in measuring the quality of mental health care. Can J Psychiatry 2010;55:549-557.

12. Parry C, Plüddemann A, Bhana A. Monitoring alcohol and drug abuse trends in South Africa (19962006): Reflections on treatment demand trends. Contemporary Drug Problems 2009;36:685-703.

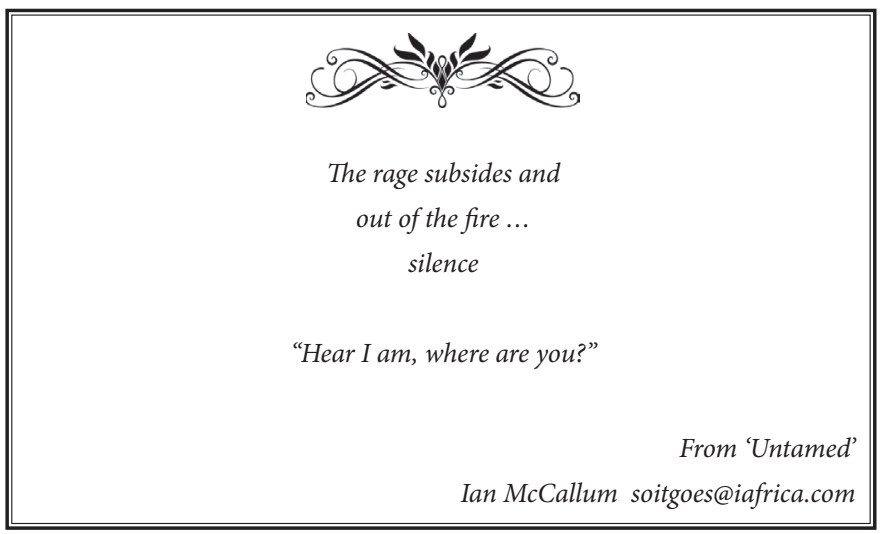

